\title{
ON THE CLUSTER VALUES OF ANALYTIC FUNCTIONS*
}

\author{
BY \\ WLADIMIR SEIDEL $\dagger$
}

INTRODUCTION

1. Let $f(z)$ be a regular, analytic function defined in the unit circle $|z|<1$. Consider a sequence of points $z_{1}, z_{2}, \cdots, z_{n}, \cdots$ lying in the interior of the unit circle and converging toward the point $z=1$, a point of discontinuity of $f(z)$. In general, the sequence of corresponding values $w_{1}=f\left(z_{1}\right), w_{2}=f\left(z_{2}\right), \cdots$, $w_{n}=f\left(z_{n}\right), \ldots$ will not converge toward a definite value. We can, however, always select a subsequence $z_{n_{1}}, z_{n_{2}}, \cdots, z_{n_{m}}, \cdots$ so that the limit of the second sequence exists:

$$
\lim _{m \rightarrow \infty} f\left(z_{n_{m}}\right)=C,
$$

where $C$ may in some cases be infinite. Such a value $C$ we shall henceforth call a cluster value of the function $f(z)$ in the point $z=1$. The set of all cluster values in the point $z=1$ we shall call the cluster set $\ddagger$ of $f(z)$ in the point $z=1$.

The purpose of the present paper is the study of the distribution of the cluster values of univalent $\S$ analytic functions, bounded analytic functions, and certain intermediate types of analytic functions in a boundary point of their circle of convergence, which without loss of generality will always be taken to be the unit circle. The point will always be taken to be $z=1$.

There are essentially three questions with which we shall be concerned. First, we shall consider cluster sets formed by taking all cluster values of $f(z)$ obtained by approaching $z=1$ along a Jordan arc and investigate certain relations between such arcs and the corresponding cluster sets. This will be done for univalent functions and for bounded functions. Secondly, we shall investigate sufficient conditions that two cluster values corresponding to two sequences of points $z_{1}, z_{2}, \cdots, z_{n}, \cdots$ and $\zeta_{1}, \zeta_{2}, \cdots, \zeta_{n}, \cdots$ of interior points of the unit circle converging towards $z=1$ be equal.

* Presented to the Society, December 30, 1930, and April 3, 1931; received by the editors July $17,1931$.

† National Research Fellow. The author wishes to express his gratitude to Professor J. D. Tamarkin for many useful suggestions.

$\ddagger$ This concept dates back to P. Painlevé, Paris Comptes Rendus, vol. 131 (1900), p. 489. points.

$\S$ By a univalent function we mean one which never assumes the same value in two different 
In the course of this investigation we shall arrive at a generalization of a theorem of W. Gross. The method used in proving it is essentially simpler than that employed by Gross. Finally, we shall consider those types of bounded functions which assume one value infinitely often in the unit circle. For these types, as will be seen, most of the preceding theorems fail to hold and we shall show alternative theorems that do hold in these cases.

The investigations that we propose to carry out are related to the work of C. Carathéodory on conformal mapping, as well as to the work of E. Lindelöf, F. Iversen, and W. Gross. ${ }^{*}$ Finally, in this connection may be mentioned an interesting recent result of A. Plessner. $\dagger$

\section{Chapter I. Cluster values on curves}

2. Let $f(z)$ be a bounded analytic function in the circle $|z|<1$;

$$
\underset{|z|<1}{\text { bound }}|f(z)|=M<\infty .
$$

Consider a Jordan $\operatorname{arc} C$ all of whose points with the exception of $z=1$ consist of interior points of the circle $|z|<1$. Joining the end points of $C$ by another

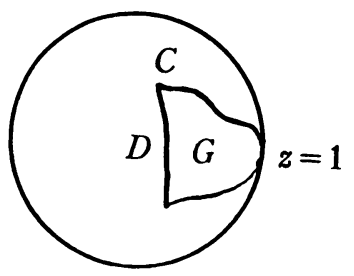

Fig. 1

Jordan $\operatorname{arc} D$ which lies in the interior of $|z|<1$ and has no points in common with $C$ except its two end points, we obtain a simply connected region $G$ bounded by the two curves $C$ and $D$ (see Fig. 1).

Let us now set

$$
\varlimsup_{z \rightarrow 1}|f(z)|=A, z \subset C .
$$

We prove the following theorem:

* C. Carathéodory, Mathematische Annalen, vol. 73 (1913), pp. 323-370; E. Lindelöf, Acta Societatis Scientiarum Fennicae, vol. 46 (1915), No. 4; F. Iversen, Paris Comptes Rendus, vol. 166 (1918), p. 156; W. Gross, Monatshefte für Mathematik und Physik, vol. 29 (1918), pp. 3-47; Mathematische Zeitschrift, vol. 2 (1918), pp. 242-294, and vol. 3 (1919), pp. 43-64.

$\dagger$ A. Plessner, Journal für Mathematik, vol. 158 (1927), pp. 219-227. 
THEOREM 1. Let $\left\{z_{i}\right\}$ be an arbitrary sequence of points lying in $G$ and converging toward $z=1$, for which

$$
\lim _{i \rightarrow \infty}\left|f\left(z_{i}\right)\right|=\alpha
$$

exists. Then $\alpha \leqq A$.

We can without loss of generality assume that the upper bound $M$ of $|f(z)|$ in the unit circle $|z|<1$ is 1 . Let $t=\phi(z)$ be the function which maps the region $G$ on the unit circle $|t|<1$ and let its inverse be $z=\psi(t)$. Since $G$

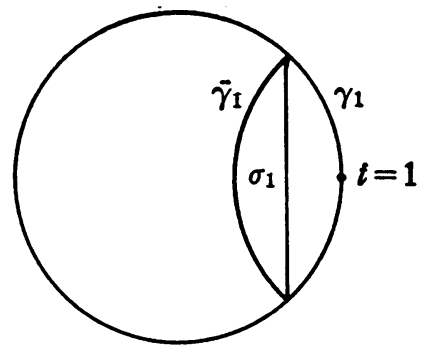

Fig. 2

is bounded by a closed Jordan curve, the function $\psi(t)$ is uniformly continuous in the closed circle $|t| \leqq 1$. $^{*}$ Furthermore, the function $\psi(t)$ can be so chosen that $\psi(1)=1$. Our map of the region $G$ carries, therefore, the sequence $\left\{z_{i}\right\}$ into a sequence of points $\left\{t_{i}\right\}$ converging toward $t=1$, for which $\lim _{i \rightarrow \infty}\left|f\left(\psi\left(t_{i}\right)\right)\right|=\alpha$. At the same time by (2.1) we have

$$
\varlimsup_{t \rightarrow 1}|f(\psi(t))|=A,|t|=1 \text {. }
$$

Let $\epsilon$ be an arbitrarily small positive constant. It follows from (2.2) that there exists an $\operatorname{arc} \gamma_{1}$ of the circumference containing the point $t=1$ such that

$$
|f(\psi(t))|<A+\epsilon
$$

for any $t$ lying on $\gamma_{1}$. Join the end points of $\gamma_{1}$ by a chord $\sigma_{1}$ and reflect the segment of the circle so formed in the chord $\sigma_{1}$ (Fig. 2). By a rigid motion of the $t$-plane we can always bring it about that the chord $\sigma_{1}$ coincides with the real axis. Let us denote the image of $\gamma_{1}$ in $\sigma_{1}$ by $\bar{\gamma}_{1}$ and the region bounded by $\gamma_{1}$ and $\bar{\gamma}_{1}$ by $\Sigma_{1}$. The function $f(\psi(t)) \bar{f}(\psi(\bar{t}))$ is analytic in $\Sigma_{1}$, and on the two $\operatorname{arcs} \gamma_{1}$ and $\bar{\gamma}_{1}$ satisfies the inequality

$$
|f(\psi(t)) \bar{f}(\psi(\bar{t}))|<A+\epsilon .
$$

* W. F. Osgood and E. H. Taylor, these Transactions, vol. 14 (1913), pp. 277-298. C. Carathéodory, Mathematische Annalen, vol. 73 (1913), pp. 305-320. 
Hence, on the chord $\sigma_{1}$ we have the inequality

$$
|f(\psi(t))|<(A+\epsilon)^{1 / 2} .
$$

Since, however, the same argument may be repeated if the chord $\sigma_{1}$ is replaced by a parallel chord lying in the region bounded by $\sigma_{1}$ and $\gamma_{1}$, we see that the inequality holds in the whole circular segment $S_{1}$ bounded by $\sigma_{1}$ and $\gamma_{1}$.

We started out by assuming that $|f(\psi(t))|<1$ in $S_{1}$ and satisfies the inequality (2.3), and arrived at the inequality (2.4) which holds in the circular segment $S_{1}$. We may again repeat the argument. Consider a chord $\sigma_{2}$ of the unit circle $|t|<1$ parallel to $\sigma_{1}$ and lying in $S_{1}$. Denoting by $\gamma_{2}$ the smaller arc subtended by $\sigma_{2}$, and by $\bar{\gamma}_{2}$ the reflection of $\gamma_{2}$ in $\sigma_{2}$, we can choose $\sigma_{2}$ in such a manner that the arc $\bar{\gamma}_{2}$ will also lie in $S_{1}$. Repeating then the argument for $\sigma_{2}$, we obtain in all points of $S_{2}$, the circular segment bounded by $\sigma_{2}$ and $\bar{\gamma}_{2}$, the inequality

$$
|f(\psi(t))|<(A+\epsilon)^{1-1 / 2^{2}} .
$$

Repeating the argument $n$ times gives for all points of the $n$th circular segment $S_{n}$

$$
|f(\psi(t))|<(A+\epsilon)^{1-1 / 2^{n}} .
$$

Since all but a finite number of the points $t_{i}$ lie in each of the segments $S_{n}$, we have

$$
\alpha=\lim _{i \rightarrow \infty}\left|f\left(\psi\left(t_{i}\right)\right)\right| \leqq \lim _{i \rightarrow \infty}(A+\epsilon)^{1-1 / 2^{n}}=A+\epsilon .
$$

Since the last inequality holds for all positive $\epsilon$, we obtain

$$
\alpha \leqq A \text {, }
$$

which completes the proof.*

3. If $f(z)$ is again taken to be the same function as in Theorem 1 , it is possible to form the following lower limit:

$$
\varliminf_{z \rightarrow 1}|f(z)|=a, z \subset C .
$$

If $\alpha$ is the same number as in Theorem 1 , it may be conjectured that $a \leqq \alpha$. An example will now be given to show that this inequality is false in general.

Consider a sequence of points $\left\{n_{k}\right\}$ lying in the interior of the unit circle $|z|<1$, converging toward $z=1$, and having the property that

$$
\prod_{k=1}^{\infty}\left|n_{k}\right|>0 \text {. }
$$

* This proof of the theorem, based on a method of Lindelöf, has been kindly suggested to me by Mr. J. Doob. 
With these numbers $\left\{n_{k}\right\}$ we form the following infinite product:*

$$
p(z)=\prod_{k=1}^{\infty} \frac{1-\frac{z}{n_{k}}}{1-\bar{n}_{k} z}\left|n_{k}\right| .
$$

It can easily be proved that when (3.2) holds, the product (3.3) converges uniformly in every bounded region $R$ of the z-plane which is at a positive distance from the points $z=1$ and $z=1 / \bar{n}_{k}$ for $k=1,2, \cdots . \dagger$

In this manner it is seen that $p(z)$ is analytic in the entire finite plane except in the points $z=1 / \bar{n}_{k}$, where it has poles, and in the point $z=1$, where it has an essential singularity. Furthermore, $|p(z)|=1$ on the circumference $|z|=1$, except in the point $z=1$, and in the interior of the circle we have $|p(z)|<1$. For the sake of completeness we shall show that $p(z)$ is analytic in the point $z=\infty$. This follows at once if we apply the reflection principle of Schwarz $\ddagger$ according to which the function may be continued analytically beyond the unit circle by means of the functional equation

$$
p\left(\frac{1}{\bar{z}}\right)=\frac{1}{\overline{p(z)}} \text {. }
$$

Hence, if the origin is not a zero of the function $p(z)$, the point $z=\infty$ is a point of analyticity.

We are now in a position to show that, if we take $f(z)=p(z)$ and consider the lower limit $a$ in equation (3.1), then the relation $a \leqq \alpha$ fails to hold always, when $\alpha$ is a cluster value of $p(z)$ in the point $z=1$. If we choose the curve to be the circumference of the unit circle itself, then we have

$$
\varliminf_{z \rightarrow 1}|p(z)|=1,|z|=1 \text {, }
$$

whereas $\alpha=0$ is certainly a cluster value of the function, for

$$
p\left(n_{k}\right)=0
$$

for all $k=1,2, \cdots$.

4. While this example, which we shall study in greater detail in the latter part of the paper, shows how complicated the distribution of cluster values

\footnotetext{
* The product (3.3) was first introduced into the theory of functions by W. Blaschke in the Leipziger Berichte, vol. 67 (1915), p. 194.

† G. Julia in his recent book, Principes Géométriques d'Analyse, Paris, 1930, p. 65, has given a simple proof of the uniform convergence of the product in every circle $|z|<\rho<1$. A simple modification of the proof shows that the convergence holds in the region $R$.

$\ddagger$ Cf. L. Bieberbach, Funktionentheorie, vol. I, p. 220.
} 
can be in the case of general types of bounded functions, we shall see that in the case of univalent functions the problem can be answered with ease.

We prove now the following theorem:

THEOREM 2. Let $f(z)$ be a univalent analytic function defined in the unit circle $|z|<1$. Let $C$ be a closed Jordan curve which passes through the point $z=1$ and save for that point lies in the interior of the unit circle. Let $\left\{z_{i}\right\}$ be a sequence of points lying in the region $G$ bounded by the curve $C$ and converging toward $z=1$. Let the limit $\lim _{i \rightarrow \infty} f\left(z_{i}\right)$ exist and be equal to $\alpha$ and let $S$ be the cluster set of $f(z)$ in the point $z=1$ assumed along $C$. Then, the point $\alpha$ lies in the set $S$.

It is clear, first of all, that we may restrict our attention to the case of univalent and bounded functions. For if $w=f(z)$ is a general univalent function, it maps the unit circle conformally on a simply connected region $R$ of the $w$-plane. This region $R$ must have at least two boundary points $w=a$ and $w=b$. $^{*}$

It can be easily shown now that every simply connected region $R$ with at least two boundary points can be mapped by a function of the type

$$
w^{\prime}=\frac{1}{[(w-a) /(w-b)]^{1 / 2}-c},
$$

where $c$ is a suitable constant, on a bounded and simply connected region $R^{\prime} \dagger$ The function

$$
F(z)=\frac{M}{[(f(z)-a) /(f(z)-b)]^{1 / 2}-c}
$$

is, therefore, univalent and bounded: $|F(z)|<1$ in the circle, mapping the circle on a region $R^{\prime \prime}$, when the constant $M$ is suitably chosen.

Suppose Theorem 2 were proved for the case of univalent and bounded functions. We shall then prove it for general univalent functions. Solving equation (4.1) for $f(z)$ yields

$$
f(z)=\frac{b(M+c F(z))^{2}-a[F(z)]^{2}}{(M+c F(z))^{2}-[F(z)]^{2}} .
$$

Let the cluster set of $F(z)$ along $C$ be $S^{\prime}$ and $\lim _{i \rightarrow \infty} F\left(z_{i}\right)=\alpha^{\prime}$. According to our assumption $\alpha^{\prime}$ lies in the set $S^{\prime}$, and we wish to show that in that case $\alpha$ will also lie in $S$. By equation (4.1) there corresponds to $\alpha$ one and only one cluster value $\alpha^{\prime}$ of $F(z)$ which lies in the set $S^{\prime}$. By (4.2), then, $\alpha$ lies in $S$.

* For a proof of this see L. Bieberbach, Funktionentheorie, vol. II, Berlin, 1931, p. 5.

$\dagger$ L. Bieberbach, loc. cit., vol. II, p. 6. 
We are justified, therefore, in proving Theorem 2 by assuming that the function $f(z)$ is univalent and bounded:

$$
|f(z)|<1
$$

Let $z=\phi(t)$ be a function which maps the region $G$, bounded by the closed Jordan curve $C$, conformally on the circle $|t|<1$ in such a manner that $z=1$ corresponds to $t=1$. By a well known theorem of Osgood and Carathéodory, mentioned on page $3, \phi(t)$ is continuous in the closed circle $|t| \leqq 1$. This function transforms the points $z_{1}, z_{2}, \cdots, z_{n}, \cdots$ into a set of points $t=t_{1}$, $t=t_{2}, \cdots, t=t_{n}, \cdots$ which lie in the interior of the unit circle $|t|<1$ and converge toward $t=1$. On this set of points the function $f(\phi(t))=\psi(t)$, which, except for $t=1$, is analytic and bounded everywhere in the circle $|t| \leqq 1$, converges toward $\alpha: \lim _{n \rightarrow \infty} \psi\left(t_{n}\right)=\alpha$. The cluster set of $\psi(t)$ in $t=1$ assumed along the circumference $|t|=1$ is $S$, the set defined by us in the statement of Theorem 2. We wish to show that $\alpha$ is contained in $S$ or, what is tantamount, that there exists a set of points $T_{1}, T_{2}, \cdots$ lying on $|t|=1$ and converging toward $t=1$ such that

$$
\lim _{n \rightarrow \infty} \psi\left(T_{n}\right)=\alpha .
$$

This last assertion may be proved without difficulty by the use of Carathéodory's theory of prime ends.* By Carathéodory's fundamental result we know that the cluster set of $\psi(t)$ in every point of $|t|=1$ is precisely a prime end of the region $R$ into which the circle $|t|<1$ is mapped by the function $\psi(t)$. In particular, to the point $t=1$ there must correspond a prime end $E$ of the region $R$. Since $\psi(t)$ is analytic elsewhere on the circumference $|t|=1$, all remaining prime ends of $R$ are single points. Thus our assertion about the existence of points $T_{1}, T_{2}, \cdots$ with the above-mentioned properties and satisfying equation (4.3) reduces geometrically to the assertion that there always exists a sequence of boundary points of $R$, not belonging to the prime end $E$, which converges toward any preassigned point $\alpha$ of $E$.

If this were not the case, there would exist a point $\alpha$ of $E$ which would not be a limit point of any sequence of boundary points of $R$ not belonging to $E$. We could then draw a circle $C$ with $\alpha$ as center and with a radius so small that every boundary point of $R$, not belonging to $E$, would lie outside of $C$. Since, however, $\alpha$, as a point of a prime end $E$, is also a boundary point of $R$, there exists a sequence of interior points of $R$ which converges toward $\alpha$. There must surely exist at least one interior point $p$ of $R$ which also lies in the interior of $C$. Consider now the line segment $\overline{p \alpha}$. Setting out from $p$ and travelling

\footnotetext{
* For a complete presentation of the theory see C. Carathéodory, Mathematische Annalen, vol.
} 73 (1913), pp. 321-370. 
along this line segment, there will exist a first boundary point $q$ of $R$ which will be reached. This is true because the boundary of $R$ is a closed point set. Since $q$ lies on the line segment between $p$ and $\alpha$, it surely lies in $C$ and is therefore a point of the prime end $E$. Moreover, $q$ is an accessible point of the prime end $E$.

If there exists a second point $\beta$ of $E$ in the circle $C$ which does not lie on the straight line through $p$ and $\alpha$, we can join $p$ and $\beta$ by a line segment $\overline{p \beta}$ and, repeating the argument of the preceding paragraph, arrive at the existence of a second accessible point $r$ of $E$, different from $q$. This, however, is a contradiction, for according to a theorem of Carathéodory* a prime end can contain at most one accessible point.

The only remaining alternative, then, is that the subset $E^{\prime}$ of $E$ which lies in the circle $C$ lies wholly on the line segment joining $p$ and $\alpha$. Since $E$ is a perfect connected set, $\uparrow E^{\prime}$ must itself be a line segment. Let us consider two points $\alpha$ and $\beta$ of $E$ lying in $C$. Not every interior point of $R$, contained in $C$, will lie on the straight line which contains $E^{\prime}$. For if $p$ is such a point, we can construct a circle $\Gamma$ about $p$ as a center and with a radius so small that $\Gamma$ will consist only of interior points of $R$ and will lie wholly within $C$. We, therefore, merely need to choose any point $P$ of $\Gamma$ which does not lie on the line containing $E^{\prime}$. Join $P$ with $\alpha$ and $\beta$ respectively, by the line segments $L_{\alpha}$ and $L_{\beta}$. Since $P$ does not lie on the line containing $E^{\prime}$, the points $\alpha$ and $\beta$ will be the first boundary points of $E$ attained by travelling from $P$ along $L_{\alpha}$ and $L_{\beta}$ respectively. Hence, $\alpha$ and $\beta$ are again two distinct accessible points of the prime end $E$, which is a contradiction.

This means that to every point $\alpha$ of a prime end $E$ there exists a sequence $\left\{p_{n}\right\}$ of boundary points of $R$, not belonging to $E$, which converges toward $\alpha$ as a limit point. Since $\psi(t)$ was taken to be analytic everywhere on the circumference of the circle $|t|<1$ except in the point $t=1$, the result means that there exists a sequence $\left\{T_{n}\right\}$ of points on the circumference $|t|=1$ converging toward $t=1$ and such that

$$
\lim _{n \rightarrow \infty} \psi\left(T_{n}\right)=\alpha .
$$

The function $\psi(t)$ was defined as $f(\phi(t))$, where $\phi(t)$ is continuous in the closed circle $|t| \leqq 1$. Hence, $\psi\left(T_{n}\right)=f\left(Z_{n}\right)$, where $\left\{Z_{n}\right\}$ is a sequence of points on $C$ converging toward $z=1$ and such that

$$
\lim _{n \rightarrow \infty} f\left(Z_{n}\right)=\alpha \text {. }
$$

This proves that $\alpha$ lies in $S$, and therewith the theorem.

* C. Carathéodory, loc. cit., p. 353.

† C. Carathéodory, loc. cit., p. 335. 


\section{Chapter II. Conditions that cluster values \\ ON TWO SEQUENCES BE EQUAL}

5. In this section we shall investigate the second problem proposed in the Introduction. Let $f(z)$ be a bounded analytic function:

$$
|f(z)|<1
$$

in the unit circle $|z|<1$. Let $z=1$ be a point of discontinuity of $f(z)$ and let $z_{1}, z_{2}, \cdots$ and $z_{1}^{\prime}, z_{2}^{\prime}, \cdots$ be two sequences of interior points of the circle converging toward $z=1$. If the limit

$$
\lim _{n \rightarrow \infty} f\left(z_{n}\right)=\alpha
$$

exists, what conditions are to be imposed upon the second sequence in order that

should also hold?

$$
\lim _{n \rightarrow \infty} f\left(z_{n}^{\prime}\right)=\alpha
$$

Before attempting to give an answer to this question, we shall briefly recall some facts about the non-euclidean interpretation of a lemma of Schwarz. If we set $w=f(z)$ and consider the $w$-plane, we see that the inequality (5.1) means that $f(z)$ only assumes such values in the circle $|z|<1$ as lie in the interior of the circle $|w|<1$. Let us consider the two circles $|z|<1$ and $|w|<1$ as carriers of a non-euclidean (Lobachevskian) geometry. In this geometry angles are measured in the same manner as in euclidean geometry, but the distance between two points $z_{1}$ and $z_{2}$ is defined as follows: Join the points $z_{1}$ and $z_{2}$ by a circle orthogonal to the unit circle $|z|<1$ and cutting it in the points $\zeta_{1}$ and $\zeta_{2}$. The non-euclidean distance $D\left(z_{1}, z_{2}\right)$ is defined by the relation

$$
D\left(z_{1}, z_{2}\right)=\log \left(\frac{z_{1}-\zeta_{1}}{z_{1}-\zeta_{2}} \frac{z_{2}-\zeta_{2}}{z_{2}-\zeta_{1}}\right)
$$

where the expression in parentheses is the ordinary cross ratio of the four

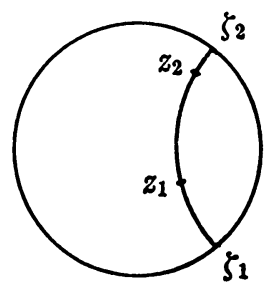

Fig. 3

points $\zeta_{1}, \zeta_{2}, z_{1}, z_{2}$ (Fig. 3). An equivalent expression, involving only $z_{1}$ and $z_{2}$, is 


$$
D\left(z_{1}, z_{2}\right)=\log \frac{1+\left|\frac{z_{1}-z_{2}}{1-\bar{z}_{1} z_{2}}\right|}{1-\left|\frac{z_{1}-z_{2}}{1-\bar{z}_{1} z_{2}}\right|} .
$$

The non-euclidean interpretation* of the Lemma of Schwarz may now be expressed in the following manner:

LEMMA OF SchWARz. Let $f(z)$ be a bounded analytic function:

$$
|f(z)|<1
$$

in the unit circle $|z|<1$ and $z_{1}$ and $z_{2}$ two interior points of the circle. If $w_{1}=f\left(z_{1}\right)$ and $w_{2}=f\left(z_{2}\right)$, the relation

$$
D\left(w_{1}, w_{2}\right) \leqq D\left(z_{1}, z_{2}\right)
$$

subsists for all pairs of interior points $z_{1}$ and $z_{2}$ of the unit circle $|z|<1$.

6. We are now in a position to investigate the problem we set out to treat. We shall prove the following theorem:

TheOREM 3. Let $f(z)$ be a bounded analytic function:

$$
|f(z)|<1
$$

in the unit circle $|z|<1$ which omits the value $\alpha$ in the unit circle. Let $z_{1}, z_{2}, \ldots$ and $z_{1}^{\prime}, z_{2}^{\prime}, \ldots$ be two sequences of interior points of the unit circle converging toward $z=1$. If the non-euclidean distance $D\left(z_{n}, z_{n}^{\prime}\right)$ is less than a constant $M$, independent of $n$ :

$$
D\left(z_{n}, z_{n}^{\prime}\right)<M \quad(n=1,2, \cdots),
$$

and if

$$
\lim _{n \rightarrow \infty} f\left(z_{n}\right)=\alpha
$$

then also

$$
\lim _{n \rightarrow \infty} f\left(z_{n}^{\prime}\right)=\alpha \text {. }
$$

As a special case, this theorem evidently asserts that the cluster sets of a univalent function $f(z)$ along any two straight lines ending in $z=1$ are identical. Furthermore, the cluster sets along any two oricycles tangent to $|z|=1$ in the point $z=1$ are identical, and in general, the cluster sets of a univalent

* This formulation of the Lemma of Schwarz is due to G. Pick, Mathematische Annalen, vol. 77 (1916), pp. 1-6. 
function along any two curves having the same order of contact with $|z|=1$ in the point $z=1$ are identical.

In order to establish this theorem, we shall first prove two easy lemmas.

LEммa 1. Let $f(z)$ be a bounded analytic function in the unit circle $|z|<1$ :

$$
|f(z)|<1 \text {. }
$$

Let $z_{1}, z_{2}, \cdots$ and $z_{1}^{\prime}, z_{2}^{\prime}, \cdots$ be two sequences of points with the same properties as in Theorem 3. Then if

$$
\lim _{n \rightarrow \infty} f\left(z_{n}\right)=1
$$

holds, the relation

$$
\lim _{n \rightarrow \infty} f\left(z_{n}^{\prime}\right)=1
$$

also holds.

This is an almost immediate consequence of the Lemma of Schwarz. In fact, from the inequality

$$
D\left(z_{n}, z_{n}^{\prime}\right)<M
$$

follows by the Lemma of Schwarz the inequality

$$
D\left(w_{n}, w_{n}^{\prime}\right)<M,
$$

where we set $w_{n}=f\left(z_{n}\right)$ and $w_{n}^{\prime}=f\left(z_{n}^{\prime}\right)$. With this notation the equation (6.1) becomes

$$
\lim _{n \rightarrow \infty} w_{n}=1
$$

The relations (6.3) and (6.4) together yield

$$
\lim _{n \rightarrow \infty} w_{n}^{\prime}=1 \text {, }
$$

which is merely another form of (6.2).

LEMma 2. Let $f(z)$ be a bounded analytic function in the unit circle $|z|<1$ :

$$
|f(z)|<1 \text {. }
$$

Let $z_{1}, z_{2}, \cdots$ and $z_{1}^{\prime}, z_{2}^{\prime}, \cdots$ be two sequences of points with the same properties as in Theorem 3. Let

$$
\phi(z)=\frac{f(z)+1}{f(z)-1} .
$$

If

$$
\lim _{n \rightarrow \infty} \Re \phi\left(z_{n}\right)=-\infty \text {, then also } \lim _{n \rightarrow \infty} \Re \phi\left(z_{n}^{\prime}\right)=-\infty \text {. }
$$


Using the notation $f(z)=u+i v$ and $\phi(z)=\xi+i \eta$ and setting the real and imaginary parts of both sides of equation (6.5) equal to each other, respectively, we obtain the equations

$$
u=\frac{\xi^{2}+\eta^{2}-1}{(\xi-1)^{2}+\eta^{2}}, \quad v=-\frac{2 \eta}{(\xi-1)^{2}+\eta^{2}} .
$$

Setting $f\left(z_{n}\right)=u_{n}+i v_{n}, \phi\left(z_{n}\right)=\xi_{n}+i \eta_{n}, f\left(z_{n}^{\prime}\right)=u_{n}^{\prime}+i v_{n}^{\prime}, \phi\left(z_{n}^{\prime}\right)=\xi_{n}^{\prime}+i \eta_{n}^{\prime}$, we have by hypothesis $\lim _{n \rightarrow \infty} \xi_{n}=-\infty$. We furthermore observe that $\xi<0$ for all points of the unit circle $|z|<1$. Solving the equations (6.6) for $\xi$ and $\eta$, we obtain the new pair of equations

$$
\xi=\frac{u^{2}+v^{2}-1}{(u-1)^{2}+v^{2}}, \quad \eta=-\frac{2 v}{(u-1)^{2}+v^{2}},
$$

as well as

$$
\xi_{n}=\frac{u_{n}^{2}+v_{n}^{2}-1}{\left(u_{n}-1\right)^{2}+v_{n}^{2}}, \eta_{n}=-\frac{2 v_{n}}{\left(u_{n}-1\right)^{2}+v_{n}^{2}} .
$$

Since $\xi_{n}<0$, the first equation (6.8) may be written in the form

$$
\left(u_{n}-\frac{\xi_{n}}{\xi_{n}-1}\right)^{2}+v_{n}^{2}=\frac{1}{\left(\xi_{n}-1\right)^{2}} .
$$

Equation (6.9) is merely the equation of a circle in the $\left(u_{n}, v_{n}\right)$-plane with center in the point $\left(\xi_{n} /\left(\xi_{n}-1\right), 0\right)$ and with a euclidean radius of length $-1 /\left(\xi_{n}-1\right)$. This circle lies in the interior of the unit circle $u_{n}^{2}+v_{n}^{2}=1$ and is tangent to this circle in the point $(1,0)$. Such a circle is called an oricycle in non-euclidean geometry. Equation (6.9) allows us to interpret geometrically the fact that $\lim _{n \rightarrow \infty} \xi_{n}=-\infty$. We find that the necessary and sufficient condition that $\lim _{n \rightarrow \infty} \xi_{n}=-\infty$ hold is that the points $\left(u_{n}, v_{n}\right)$ lie on oricycles tangent to $u_{n}^{2}+v_{n}^{2}=1$ in the point $(1,0)$ whose euclidean radii converge toward zero.

From the inequality $D\left(z_{n}, z_{n}^{\prime}\right)<M$ follows the inequality $D\left(u_{n}+i v_{n}\right.$, $\left.u_{n}^{\prime}+i v_{n}^{\prime}\right)<M$ by Schwarz's Lemma. Since the points $u_{n}+i v_{n}$ lie on oricycles which converge toward zero, the points $u_{n}^{\prime}+i v_{n}^{\prime}$ must also lie on oricycles converging toward zero. This means, according to the remark made at the end of the last paragraph, that $\lim _{n \rightarrow \infty} \xi_{n}^{\prime}=-\infty$, which proves our second lemma.

We can now proceed with the proof of Theorem 3 . There are two cases to be considered, according as $|\alpha|=1$ or $|\alpha|<1$. If $|\alpha|=1$, we set $\phi(z)=\bar{\alpha} f(z)$. We then have 


$$
\lim _{n \rightarrow \infty} \phi\left(z_{n}\right)=1
$$

According to Lemma 1, whose hypotheses are satisfied by $\phi(z)$, we also have

$$
\lim _{n \rightarrow \infty} \phi\left(z_{n}^{\prime}\right)=1 \text {, }
$$

or

$$
\lim _{n \rightarrow \infty} f\left(z_{n}^{\prime}\right)=\alpha,
$$

which proves the proposition.

If $|\alpha|<1$, we introduce the transformation

$$
\phi(z)=\frac{f(z)-\alpha}{1-\bar{\alpha} f(z)}
$$

which defines a bounded analytic function $\phi(z)$ in the unit circle: $|\phi(z)|<1$ in $|z|<1$, for which

$$
\lim _{n \rightarrow \infty} \phi\left(z_{n}\right)=0
$$

and which omits the value 0 in the circle. We choose an arbitrary one of the branches of $\log \phi(z)$, each of which is a single-valued function in the unit circle. Furthermore, this function, which we denote by $\psi(z)$, satisfies the relation

$$
\Re \psi(z)=\log |\phi(z)|<0
$$

and

$$
\lim _{n \rightarrow \infty} \Re \psi\left(z_{n}\right)=-\infty
$$

Finally, we consider the function

$$
\chi(z)=\frac{\psi(z)+1}{\psi(z)-1} .
$$

This function is again analytic in the unit circle $|z|<1$ and is bounded there: $|\chi(z)|<1$. By Lemma 2, we have then the relation

$$
\lim _{n \rightarrow \infty} \Re \psi\left(z_{n}^{\prime}\right)=-\infty
$$

or according to (6.11)

$$
\lim _{n \rightarrow \infty} \phi\left(z_{n}^{\prime}\right)=0
$$


From this and equation (6.10) follows $\lim _{n \rightarrow \infty} f\left(z_{n}^{\prime}\right)=\alpha$, which completely proves our theorem.*

As a final remark, we observe that Theorem 3 holds if we allow $f(z)$ to assume the value $\alpha$ a finite number of times in the circle. For in that case there always exists a neighborhood $N$ of the point $z=1$ in which the function $f(z)$ does not assume the value $\alpha$. By a conformal map of the region $N$ on a circle we reduce the situation to that of Theorem 3 .

If, however, $f(z)$ assumes the value $\alpha$ in every neighborhood of $z=1$, which is only possible if the value is assumed infinitely often, then it may be shown by an example that the theorem fails to be true. Such an example will now be constructed.

7. The example we shall consider shows that if a function has infinitely many zeros in the unit circle, Theorem 3 may fail to hold.

Let $0<t_{1}<t_{2}<\cdots<t_{n}<\cdots$ be a sequence of real, positive numbers less than one and converging toward one: $\lim _{n \rightarrow \infty} t_{n}=1$. Let these numbers be chosen in such a manner that

$$
\prod_{n=1}^{\infty} t_{n}>0 .
$$

Then, it is well known that the product

$$
\phi(z)=\prod_{n=1}^{\infty} \frac{z-t_{n}}{1-t_{n} z}
$$

represents a bounded analytic function in the unit circle: $|\phi(z)|<1$ with zeros in the points $z=t_{n}(n=1,2, \cdots)$. In particular, if we set

$$
t_{n}=\frac{n !-1}{n !+1} \quad(n=1,2, \cdots),
$$

(7.1) is satisfied. We wish to show that Theorem 3 fails to hold for this particular $\phi(z)$. In order to show this, we consider a second sequence

$$
\tau_{n}=\frac{(n+1) !-\rho}{(n+1) !+\rho}, 1<\rho<2 \quad(n=1,2, \cdots),
$$

of real positive numbers less than one and converging toward one:

$$
\lim _{n \rightarrow \infty} \tau_{n}=1 \text {. }
$$

Furthermore, the non-euclidean distance

$$
D\left(\tau_{n}, t_{n+1}\right)=\log \rho
$$

* This theorem is a generalization of a theorem of W. Gross, Monatshefte für Mathematik und Physik, vol. 29 (1918), pp. 3-47. 
is bounded for all values of $n$, and, as we shall prove,

$$
\lim _{n \rightarrow \infty}\left|\phi\left(\tau_{n}\right)\right|=\frac{\rho-1}{\rho+1}>0 .
$$

Setting $z=\tau_{j}$ in equation (7.2) and substituting there the values of $t_{n}$ and $\tau_{i}$, as given in equations (7.3) and (7.4), respectively, we obtain

$$
\phi\left(\tau_{i}\right)=\prod_{n=1}^{\infty} \frac{(j+1) !-\rho n !}{(j+1) !+\rho n !} .
$$

Hence,

(7.8) $\left|\phi\left(\tau_{j}\right)\right|=\prod_{n=1}^{j-1} \frac{(j+1) !-\rho n !}{(j+1) !+\rho n !} \prod_{n=i}^{j+2}\left|\frac{(j+1) !-\rho n !}{(j+1) !+\rho n !}\right| \prod_{n=j+3}^{\infty} \frac{\rho n !-(j+1) !}{\rho n !+(j+1) !}$.

The second factor obviously tends to $(\rho-1) /(\rho+1)$ as $j \rightarrow \infty$. We shall show now that the limits of the first and third factors are 1 as $j$ becomes infinite.

Consider the first factor in the form

$$
\exp \left[\sum_{n=1}^{j-1} \log \frac{(j+1) !-\rho n !}{(j+1) !+\rho n !}\right] \text {. }
$$

Here

$\log \frac{(j+1) !-\rho n !}{(j+1) !+\rho n !}=O\left[\log \left(1+\frac{1}{j^{2}}\right)\right]=O\left(j^{-2}\right) \quad(n=1,2, \cdots, j-1)$.

The exponent in (7.9) is

$$
(j-1) O\left(j^{-2}\right)=O\left(j^{-1}\right) \rightarrow 0
$$

as $j \rightarrow \infty$. As to the last factor in (7.8),

$$
\exp \left[\sum_{n=j+3}^{\infty} \log \frac{\rho n !-(j+1) !}{\rho n !+(j+1) !}\right]
$$

we have

$$
\log \frac{\rho n !-(j+1) !}{\rho n !+(j+1) !}=O\left(n^{-2}\right),
$$

whence the exponent in $(7.10)$ is

$$
O\left(\sum_{n=j+3}^{\infty} n^{-2}\right)=O\left(j^{-1}\right) \rightarrow 0
$$

as $j \rightarrow \infty$. This proves (7.6). 
Chapter III. Cluster values of bounded functions

8. The example given in $\S 7$ shows that bounded functions assuming one value infinitely often may have entirely different properties as regards their cluster values. It is the study of these properties that we turn to now.

We return to the function studied in $\S 3$ :

$$
p(z)=\prod_{k=1}^{\infty} \frac{1-\frac{z}{n_{k}}}{1-\bar{n}_{k} z}\left|n_{k}\right|, n_{k} \rightarrow 1,
$$

where the product $\Pi_{k=1}^{\infty}\left|n_{k}\right|>0$. This function, as we have seen, is analytic in the whole $z$-plane, with the exception of the points $z=1 / \bar{n}_{k}(k=1,2, \cdots)$, where $p(z)$ has poles, and the point $z=1$ which is an isolated essential singularity and limit point of poles.

In accordance with the Picard theorem the function $p(z)$ assumes in every neighborhood of the point $z=1$ every value infinitely often with the exception of at most two values. We possess, however, additional information as to the distribution of the values. The general factor

$$
\left|n_{k}\right| \frac{1-\frac{z}{n_{k}}}{1-\bar{n}_{k} z}
$$

of the product $p(z)$ satisfies the inequality

$$
\left|n_{k} \frac{1-\frac{z}{. n_{k}}}{1-\bar{n}_{k} z}\right| \geqq 1
$$

in $|z| \geqq 1$. Hence, the inequality $|p(z)| \geqq 1$ holds also in $|z| \geqq 1$, and only in $|z| \geqq 1$. Hence, with the exception of at most two values $a$ and $b$, every value $\alpha,|\alpha|<1$, is assumed infinitely often by $p(z)$ in points of the unit circle $|z|<1$ which converge toward the point $z=1$. It can be shown, moreover, that at most one value $a,|a|<1$, can be omitted (or assumed only a finite number of times). For if $a$ is such an exceptional value, then according to the relation (3.4):

$$
p\left(\frac{1}{\bar{z}}\right)=\frac{1}{\overline{p(z)}},
$$

which may also be directly verified in the product (8.1), the value $1 / \bar{a}$ will also be exceptional. If, now, a second value $b,|b|<1$, were also omitted, then there would be three values omitted (or only assumed a finite number of 
times) in the neighborhood of $z=1$. Hence, we have that with the exception of at most one value $a,|a|<1$, every value $\alpha,|\alpha|<1$, is assumed infinitely often by $p(z)$ in points of the unit circle $|z|<1$ which converge toward the point $z=1$, and no value $\beta,|\beta| \geqq 1$, is assumed by $p(z)$ in a point of the unit circle $|z|<1$.

If instead of Picard's Theorem, we had used Weierstrass' theorem that in the neighborhood of an isolated essential singularity a function approaches every preassigned value, and then applied the same reasoning as before, we would find that the cluster set of the function $w=p(z)$ in the point $z=1$ is the closed unit circle $|w| \leqq 1$.

9. From the function $p(z)$ studied in $\$ 8$ we immediately obtain an interesting example. Let $G$ be an arbitrary simply connected region lying in the interior of the unit circle $|w|<1$. A function $w=F(z)$ will be constructed which is analytic and bounded in the unit circle $|z|<1$ :

$$
|F(z)|<1
$$

and whose cluster set in the point $z=1$ is precisely the closed cover $\bar{G}$ of the region $G$. Furthermore, every value, save at most one, out of the region $G$ will be assumed by the function $F(z)$ infinitely often in the circle $|z|<1$.

Let $w=\phi(t)$ be a function which maps the region $G$ on the unit circle $|t|<1$ in the $t$-plane. The function $F(z)$ in question will be given by

$$
F(z)=\phi[p(z)] .
$$

10. The behavior exemplified by the function $p(z)$ is characteristic of a wider class of functions. We prove the following theorem:

THEOREM 4. Let $w=f(z)$ be a bounded analytic function in the unit circle $|z|<1:|f(z)|<1$. Let $\left\{n_{k}\right\}$ be an infinite sequence of points interior to the unit circle converging toward $z=1$ in which the function vanishes and let $A$ be an arc of the unit circle, $-\alpha \leqq \theta \leqq \alpha, z=e^{i \theta}$, containing $z=1$, on which $f(z)$ is continuous except for $z=1$ and assumes values of modulus 1 . Then, $w=f(z)$ assumes every value $w$, save at most one, of the unit circle $|w|<1$ infinitely often in the unit circle $|z|<1$ and assumes no value $w,|w| \geqq 1$, in the unit circle. The cluster set of $f(z)$ in $z=1$ is the closed unit circle $|w| \leqq 1$.

The proof of this theorem is practically the same as in $\S 8$. We only have to extend $f(z)$ analytically across the arc $A$ by means of the functional equation

$$
f\left(\frac{1}{\bar{z}}\right)=\frac{1}{\overline{f(z)}} .
$$


11. By conformal mapping Theorem 4 is immediately extended as follows:

THEOREM 5. Let $w=f(z)$ be a bounded analytic function in the circle $|z|<1$, assuming values there which lie in the interior of a region $G$ bounded by a closed Jordan curve $C$. Let there be infinitely many zeros $\left\{n_{k}\right\}$ of $f(z)$ in the circle converging in the point $z=1$ and let $A$ be an arc of the circle, $-\alpha \leqq \theta \leqq \alpha, z=e^{i \theta}$, containing $z=1$, on which $f(z)$ is continuous except for $z=1$ and assumes values which all lie on the curve $C$. Then, $w=f(z)$ assumes infinitely often every value $w$, save at most one, of the region $G$, bounded by $C$, in the circle $|z|<1$. Furthermore $f(z)$, assumes no value $w$ in the circle $|z|<1$ which lies on the boundary or in the exterior of $G$. The cluster set of $f(z)$ in the point $z=1$ is the closed domain $G+C$.

12. The examples, studied thus far, of bounded functions with infinitely many zeros suggest the following alternative to Theorem 3:

THEOREM 6. Let $f(z)$ be a bounded analytic function in the unit circle $|z|<1$ :

$$
|f(z)|<1
$$

Let $z_{1}, z_{2}, \cdots$ and $z_{1}^{\prime}, z_{2}^{\prime}, \cdots$ be two sequences of interior points of the unit circle converging toward $z=1$, such that the non-euclidean distance $D\left(z_{n}, z_{n}^{\prime}\right)$ is less than a positive constant $M$, independent of $n: D\left(z_{n}, z_{n}^{\prime}\right)<M(n=1,2, \cdots)$. Then, $f(z)$ always has one of the following two properties:

I. The cluster sets of $f(z)$ on any two such sequences $\left\{z_{n}\right\}$ and $\left\{z_{n}^{\prime}\right\}$ are identical.

II. The cluster set of $f(z)$ in $z=1$ contains a circle of the w-plane. Every value from the interior of this circle is assumed infinitely many times by $f(z)$ in $|z|<1$.

If the property I fails to hold for some function $f(z)$, then there must exist a sequence $\left\{z_{n}\right\}$ of interior points of the unit circle converging toward the point $z=1$ on which the function $f(z)$ approaches a value $a$ and a second sequence $\left\{z_{n}^{\prime}\right\}$ of interior points for which the relation

$$
D\left(z_{n}, z_{n}^{\prime}\right)<M \quad(n=1,2, \cdots)
$$

holds and such that on it the function $f(z)$ approaches a value $b$, different from $a$.

Consider, now, two sets of non-euclidean circles $C_{k}$ and $D_{k}$ of radius $M+\epsilon$, $\epsilon>0$, and $M$, respectively, described about the points $z=z_{k}$ as centers. According to the relation (12.1) each circle $D_{k}$ contains the corresponding point $z_{k}^{\prime}$ in its interior and each circle $D_{k}$ is contained in the interior of the corresponding $\operatorname{circle} C_{k}$. Let us now transform the circle $C_{k}$ by the transformation 


$$
z=\frac{z_{k}+m_{\epsilon} w}{1+m_{\epsilon} \bar{z}_{k} w}, \quad m_{\epsilon}=\frac{e^{M+\epsilon}-1}{e^{M+\epsilon}+1},
$$

into the unit circle $|w|<1$. The function $f(z)$ is thereby transformed into the function

$$
\psi_{k}(w)=f\left(\frac{z_{k}+m_{\epsilon} w}{1+m_{\epsilon} \bar{z}_{k} w}\right) .
$$

The functions $\psi_{k}(w)$ are defined and analytic in the circle $|w|<1$ for all $k$. Furthermore,

$$
\left|\psi_{k}(w)\right|<1 \quad(k=1,2, \cdots)
$$

and

$$
\psi_{k}(0)=f\left(z_{k}\right) \quad(k=1,2, \cdots) .
$$

The transformation (12.2) carries the circle $D_{k}$ into the circle

$$
|w| \leqq \frac{m_{0}}{m_{\epsilon}}<1
$$

Hence, the images $w_{k}^{\prime}$ of the points $z_{k}^{\prime}$ satisfy the inequality

$$
\left|w_{k}^{\prime}\right| \leqq \frac{m_{0}}{m_{s}} \quad(k=1,2, \cdots) .
$$

According to (12.5) and our assumption we have

$$
\lim _{k \rightarrow \infty} \psi_{k}(0)=a .
$$

By a well known theorem of Montel* the family $\left\{\psi_{k}(w)\right\}$, being uniformly bounded according to (12.4), forms a normal family. It is therefore possible to extract a subsequence $\left\{\psi_{k_{i}}(w)\right\}$ converging uniformly in every closed subregion of the circle $|w|<1$ which lies wholly in its interior, hence, in particular in the circle (12.6). The limit function we shall call $\chi(w)$ :

$$
\lim _{i \rightarrow \infty} \psi_{k_{i}}(w)=\chi(w) .
$$

The function $\chi(w)$ is analytic and bounded in the circle $|w|<1:|\chi(w)|<1$. From (12.8) follows

$$
\chi(0)=a
$$

* See P. Montel, Leçons sur les Familles Normales, Paris, 1927, p. 21. 
In order to show that $\chi(w)$ is different from a constant it must be proved that there exists at least one point of the circle $|w|<1$ in which $\chi(w)$ is different from $a$.

Denote by $w^{\prime}$ an arbitrary one of the limit points of the sequence $\left\{w_{k_{i}}^{\prime}\right\}$. By (12.7) it follows that all points as well as all their limit points lie in the circle (12.6). Since the convergence (12.9) in that circle is uniform, it follows that to each arbitrary positive $\eta$ there exists a positive integer $k(\eta)$, independent of $w$, such that

$$
\left|\chi\left(w_{k_{i}}^{\prime}\right)-\psi_{k_{i}}\left(w_{k_{i}}^{\prime}\right)\right|<\frac{\eta}{2} \text { for } k_{i}>k(\eta) .
$$

Furthermore, it follows from the uniform continuity of $\chi(w)$ in the circle (12.6) that to the given $\eta$ there corresponds a positive integer $K(\eta)$, independent of $w$, such that

$$
\left|\chi\left(w_{k_{i}^{\prime}}^{\prime}\right)-\chi\left(w^{\prime}\right)\right|<\frac{\eta}{2} \text { for } k_{i}>K(\eta) .
$$

Adding together the inequalities (12.10) and (12.11) yields

$$
\left|\chi\left(w^{\prime}\right)-\psi_{k_{i}}\left(w_{k_{i}}^{\prime}\right)\right|<\eta
$$

for $k_{i}>k(\eta)$ and $k_{i}>K(\eta)$, or

$$
\lim _{i \rightarrow \infty} \psi_{k_{i}}\left(w_{k_{i}^{\prime}}^{\prime}\right)=\chi\left(w^{\prime}\right)
$$

From the last equation and (12.3) it follows that

$$
\chi\left(w^{\prime}\right)=\lim _{i \rightarrow \infty} f\left(z_{k_{i}}^{\prime}\right)=b,
$$

which proves that $\chi(w)$ is not a constant.

Hence, there exists a circle $\left|t-t_{0}\right|<\gamma$ such that the function $t=\chi(w)$ assumes in the circle (12.6) every value of the circle $\left|t-t_{0}\right|<\gamma$ at least once.

It will be shown now that every value $t$ assumed by the function $\chi(w)$ in the circle $|w|<1$ is a cluster value of the function $f(z)$ in the point $z=1$. Let $t$ be an arbitrary such value assumed by $\chi(w)$ in some point $w_{0}$ of the unit circle. Consider the numbers

$$
\psi_{k_{i}}\left(w_{0}\right)=t_{k_{i}}
$$

Let the image point of $w_{0}$ by the transformation (12.2) be denoted by $\zeta_{k}$. Then, the point $z=\zeta_{k_{i}}$ is a point of the circle $C_{k_{i}}$ and we have by (12.3)

$$
f\left(\zeta_{k_{i}}\right)=t_{k_{i}}
$$


Now $\lim _{i \rightarrow \infty} t_{k_{i}}=t$ by (12.9) and (12.12). Hence

$$
\lim _{i \rightarrow \infty} f\left(\zeta_{k_{i}}\right)=t
$$

as we set out to prove. Hence, the cluster set of $f(z)$ in the point $z=1$ contains the circle $\left|t-t_{0}\right|<\gamma$. The last statement of the theorem follows immediately if we observe that, in view of the uniform convergence of the sequence $\left\{\psi_{k_{i}}(w)\right\}$ to $\chi(w)$ in the interior of $|w|<1$, the equations $\chi(w)=t, \psi_{k_{i}}(w)=t$ have the same number of roots for sufficiently large values of $i, t$ being fixed.*

HARVARD UNIVERSTTY,

Cambrimge, Mass.

* P. Montel, Leçons sur les Familles Normales, Paris, 1927, p. 20. 